\title{
Low molecular weight nucleoside gelators: A platform for protein aggregation inhibition
}

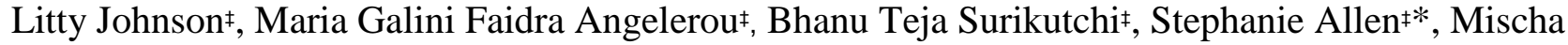 \\ Zelzer ${ }^{*}$ and Maria Marlow ${ }^{*}$
}

‡School of Pharmacy, University of Nottingham, Nottingham NG72RD, United Kingdom.

Supporting Information Placeholder

\begin{abstract}
Low molecular weight nucleoside gelators hold great promise in drug delivery and particularly for the delivery of biologics due to their excellent biocompatibility. However, the influence of these gelators on protein aggregation inhibition has not yet been studied. Protein aggregation is the most significant cause of protein instability and can severely impact the biological activity of the protein, impairing the quality and safety of the formulation. Herein, we report the ability of a nucleoside based gelator, N4-octanoyl-2'-deoxycytidine to inhibit protein aggregation. Using turbidimetric, spectroscopic and microscopic methods, we demonstrate that protein aggregation inhibition is dependent on gelator concentration. Moreover, we have found that the protein is still functionally active in the hydrogel.
\end{abstract}

Keywords: Protein aggregation inhibition, Nucleoside gelator, Gel

Protein and peptide pharmaceuticals are one of the most rapidly growing class of clinical therapeutics, ${ }^{1-2}$ where they have a significant therapeutic role in cancer, inflammatory diseases, neurological disorders and vaccines. ${ }^{3-4}$ Despite their importance, the application of protein and peptide therapeutics is limited by the propensity of these molecules to form aggregates. These aggregates can severely impact the biological activity of the peptide/protein, impairing the quality and safety of the formulation and hence reducing the efficacy of the therapeutic. ${ }^{5}$ Hence, a key challenge in the biopharmaceutical industry is the development of a stable protein formulation that minimizes or prevents protein aggregation. ${ }^{6}$

Currently, the main strategy used to inhibit protein aggregation is the addition of solute excipients and/or co-solvents (stabilizers) such as glycerol ${ }^{7}$, arginine hydrochloride ${ }^{8}$, polysorbate $80^{9}$ and others to the protein solution. ${ }^{10}$ While some compounds such as glycine are well-known to stabilise antibodies ${ }^{11}$, they also have the drawback of destabilizing other proteins such as myoglobin. Thus, the action of stabilisers often depends on the nature of the target protein and hence the choice of an effective stabiliser and selection of the stabiliser concentration for a specific protein can be an exhaustive process. ${ }^{12-13}$ In addition, large amounts of stabilizers can be required to obtain the desired effect and this may increase the cost of the formulation or potentially lead to toxicity.

Thus, there is an urgent need for the development of novel methods to prevent protein aggregation which may be applicable to many proteins at low concentrations and which are also relatively economic, biocompatible and biodegradable. Hence, herein we describe for the ability of a supramolecular, low molecular weight gelator (LMWG) based hydrogel formulation to prevent protein aggregation. Others in the field have demonstrated the ability of proteins to enhance the order of self-assembled supramolecular hydrogels ${ }^{14}$; prevention of protein denaturisation with these supramolecular hydrogels ${ }^{15}$ and finally their capability to immobilize proteins through interaction with the hydrophobic core of their nanofibers ${ }^{16-}$ ${ }^{19}$; however, inhibition of protein aggregation in the presence of a supramolecular hydrogel has not yet been reported.

LMWGs, particularly peptide based molecules, have been evaluated as platforms for protein delivery but not as an approach for the prevention of protein aggregation. ${ }^{20-21}$ A peptide based nanofibrous hydrogel was found to be effective for delivering HIV DNA vaccines. ${ }^{22}$ Similarly an injectable two layered peptide hydrogel system was described as an approach for delivering therapeutic antibodies. ${ }^{23}$ A novel self-assembling peptide nanofiber hydrogel scaffold was also reported to be used as a carrier system for therapeutic proteins with different physical and chemical properties. ${ }^{24}$

LMWGs form hydrogels by self-assembly into a cross-linked network of nanofibers capable of retaining large amounts of water and thus forms a three dimensional structure. Solvophobic forces and $\pi-\pi$ stacking drives the self-assembly of the gelator and involves the formation of cylindrical nanofibers with a hydrophobic core. These nanofibers in the hydrogel are further stabilized by hydrogen bonding. ${ }^{25}$ Some unique properties of hydrogels such as the high water content, soft and porous nature, and biocompatibility make them ideal reservoirs for large quantities of water-soluble molecules such as peptides and proteins. ${ }^{26-27}$ In this study, we have used a nucleoside based LMWG, N4-octanoyl-2'-deoxycytidine to inhibit aggregation of the proteins lysozyme and beta-lactoglobulin.

This gelator molecule was previously developed by our group as an injectable, self-healing hydrogel ${ }^{26}$ The hydrogel system possesses the ability to shear thin in response to a stress but then reforms its structure by the formation of new interactions and hence regains its mechanical properties when the stress is removed. ${ }^{28}$ Thus the hydrogel can be effectively injected through a syringe but then will form a gel. This property makes these hydrogels ideal candidates for the delivery of biologics.

The nucleoside hydrogels were prepared by dissolving low quantities $(0.3-1.25 \% \mathrm{w} / \mathrm{v})$ of nucleoside gelator in different buffers with the aid of gentle heating and subsequent cooling to $22{ }^{\circ} \mathrm{C}$. The energy barriers for the aqueous dissolution of the gelator is overcome by the increased energy supplied by heat. Upon cooling, self-assembly into a 3D network of entangled fibers takes place 
which can entrap water molecules by capillary forces ${ }^{29}$ and into which proteins can be incorporated. This fiber framework of cytidine-based gels was previously shown to control the release of a high molecular weight compound, i.e. dextran. ${ }^{26,30}$.

In this study, we investigated whether protein aggregation could be prevented through weak non-covalent interactions between the protein and the gelator. To investigate this hypothesis we chose two model proteins, lysozyme and beta-lactoglobulin, that are well known to aggregate under various conditions. ${ }^{31}$ These model proteins at the concentration of $3 \% \mathrm{w} / \mathrm{v}$ were incorporated into hydrogels prepared in phosphate buffered saline (pH 7.4) at 37 ${ }^{\circ} \mathrm{C}$ and then allowed to cool at $22{ }^{\circ} \mathrm{C}$. These hydrogels with lysozyme and beta-lactoglobulin at different concentrations of gelator in phosphate buffered saline are shown in ESI, Figure S1. The gelator formed viscoelastic gels stable to vial inversion at physiological $\mathrm{pH}$ in the presence of both proteins. The gels were homogenously turbid consistent with previous data. ${ }^{26}$ Oscillatory rheology was used to confirm the viscoelastic nature of the protein containing hydrogels (ESI -Figures S9) where the storage and loss moduli $\left(G^{\prime}\right.$ and $\left.G^{\prime \prime}\right)$ were similar to previous reported values for the gelator without protein at $0.5 \% \mathrm{w} / \mathrm{w}\left(\mathrm{G}^{\prime}=300\right.$ and $\mathrm{G}^{\prime \prime}$ ' $=100$ $\mathrm{Pa}$ at $1 \%$ strain and a frequency of $\left.10 \mathrm{rads}^{-1}\right) .{ }^{26} \mathrm{In}$ addition, the lysozyme in the hydrogel system was found to have the same activity as that of the pure lysozyme (ESI, Figure S2).

Protein aggregation has been extensively studied and can be artificially induced by various triggers such as organic solvents, temperature or $\mathrm{pH} .{ }^{32}$ In our study, aggregation was facilitated by shifting the solution $\mathrm{pH}$ to the protein's isoelectric point, where the charge balance causes attractive forces between proteins to be predominant. ${ }^{33}$ We used previously reported conditions to induce protein aggregation i.e. $100 \mathrm{mM}$ sodium phosphate solution $\mathrm{pH} 12.2$ for lysozyme ${ }^{34}$ and $1 \mathrm{M}$ sodium chloride $\mathrm{pH} 4.6$ for beta-lactoglobulin. ${ }^{35}$ Herein, the two reported conditions for inducing protein aggregation in lysozyme and beta-lactoglobulin were successful and thus were used subsequently to study the ability of the gelator to inhibit protein aggregation.

Firstly, we assessed the ability of the nucleoside hydrogels to inhibit protein aggregation of lysozyme in sodium phosphate solution $(\mathrm{pH} 12.2)$ with a lysozyme concentration of $3 \% \mathrm{w} / \mathrm{v}$, where lysozyme has been previously reported to aggregate. ${ }^{34}$ The lysozyme aggregates in the vials were large enough to be visualised by the naked eye and could be visually compared with lysozyme in the presence of different concentrations of gelator in the same buffer. It was observed that in the presence of $0.3 \% \mathrm{w} / \mathrm{v}$ gelator, the aggregation induced turbidity was reduced when compared to lysozyme in $\mathrm{pH} 12.2$ solution alone. With increasing gelator concentrations $(1 \%$ and $1.25 \% \mathrm{w} / \mathrm{v})$, no protein aggregates were visible (Figure 1A). At $\mathrm{pH}$ 12.2, at which protein aggregation is induced, the gelator did not gel as confirmed by vial inversion (ESI, Figure S3C). This is likely due to deprotonation of the acidic ribose hydroxyl groups which has been reported for nucleosides before ${ }^{36}$, converting the gelator into an ionized state and hence causing it to remain in solution. In this condition (solution state) optical observation suggests the absence of any aggregates in the presence of gelator.

The visual observations were confirmed analytically with turbidity measurements. The turbidity of the gelator solutions (control) and solutions of gelator with lysozyme were measured at 500 $\mathrm{nm}$ as previously reported.${ }^{37-38}$ The turbidity of lysozyme is an indicator of protein aggregation as the protein does not exhibit any turbidity in physiological pH (ESI, Figure S3D). The gelator by itself showed very low levels of turbidity and hence it did not interfere with the turbidity measurement of aggregates. Indeed, it was found that for gelator samples with proteins, increasing gelator concentration led to a decrease in the turbidity of the sample. At the highest gelator concentration $(1.25 \% \mathrm{w} / \mathrm{v})$, the turbidity of the sam-
A

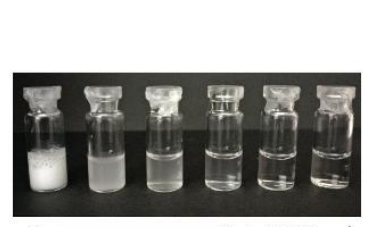

B

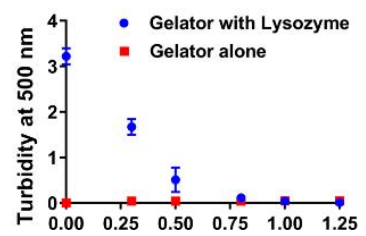

Figure 1 A: Photographs of the nucleoside gelator with lysozyme at different gelator concentrations (left to right $0,0.3$, $0.5,0.8,1.0$ and $1.25 \% \mathrm{w} / \mathrm{v})$. B: Turbidity measured at different gelator concentrations for samples containing gelator alone and gelator with $3 \% \mathrm{w} / \mathrm{v}$ lysozyme in $100 \mathrm{mM} \mathrm{pH} 12.2$ sodium phosphate solution $(\mathrm{I}=0.7 \mathrm{M})$ after mixing at $37{ }^{\circ} \mathrm{C}$ and allowing to cool at $22{ }^{\circ} \mathrm{C}$ for $15 \mathrm{~min}$.

ple was found to be nearly zero (Figure 1B). The decrease in turbidity with increasing concentration of gelator clearly demonstrates the inhibition of protein aggregation in the presence of this nucleoside gelator.

The inhibition of lysozyme aggregation in the presence of the gelator was further confirmed by optical microscopy. Optical microscopy was favoured as the lysozyme aggregates were visible to the naked eye. From the microscopy images, it was observed that the lysozyme aggregates without any gelator present showed densely packed spherical structures (ESI, Figure S4A) measuring approximately 5 to $8.8 \mu \mathrm{m}$. These amorphous aggregates were found to be larger than the aggregates reported by Kumar et al. using AFM and this is likely due to the higher concentration of proteins (5.5 times more) used here. ${ }^{34}$ These aggregates were also observed in the presence of $0.3 \%, 0.5 \%$ and $0.8 \% \mathrm{w} / \mathrm{v}$ gelator although their numbers were lower. In contrast, in samples containing $1 \%$ and $1.25 \% \mathrm{w} / \mathrm{v}$ gelator concentrations, these structures were absent suggesting that lysozyme does not aggregate under these conditions (ESI, Figure S4E and F).

The ability of the gelator to decrease or inhibit protein aggregation was further explored using an ANS (8-anilino-1-napthalene sulfonic acid) binding assay using fluorescence spectroscopy and microscopy. Upon protein aggregation, ANS is known to display an increase in its fluorescence intensity when bound to hydrophobic regions in the protein that become accessible upon aggregation. ${ }^{34}$ The fluorescence intensity of the samples and controls were recorded at the emission wavelength of $476 \mathrm{~nm}, 5$ hours after addition of ANS to allow the diffusion of ANS into the solution and its interaction with the aggregated protein. Protein samples $(\mathrm{P})$ prepared at $\mathrm{pH} 12.2$ showed increased fluorescence intensity, demonstrating that the assay is able to report the aggregation of the protein. A significantly lower fluorescence signal was observed for lysozyme at $\mathrm{pH} 12.2$ in the gelator solution $(\mathrm{G})$ (Figure 2A), consistent with a lower number of ANS binding sites for the fully folded proteins in the gelator solution. The significant difference in fluorescence intensity was demonstrated using a one way ANOVA followed by post hoc Tukey's multiple comparison test ( $\mathrm{P}$ value less than 0.0001). The control samples, of solvent/solution (S) at $\mathrm{pH} 12.2$ and 7.4 clearly indicate that the $\mathrm{pH}$ of the solution does not affect the ANS fluorescence. Further control samples of gels containing no proteins $(\mathrm{G})$ and samples of gelator with preformed protein aggregates $(\mathrm{G}+\mathrm{A})$ confirms the ability of the ANS to detect aggregated protein in the gel. This ANS fluorescence data is further confirmation of the ability of the gelator to inhibit protein aggregation. 
A
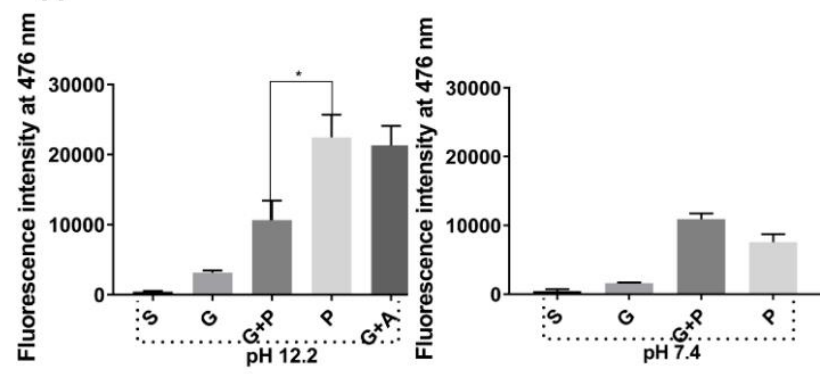

B

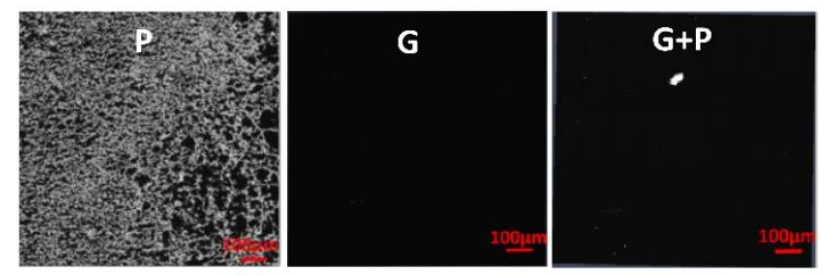

Figure 2 A: ANS Fluorescence intensities of samples and controls of lysozyme (solvent $(S)$, gelator $(\mathrm{G})$, gelator with protein $(\mathrm{G}+\mathrm{P})$, protein $(\mathrm{P})$, gelator with aggregates $(\mathrm{G}+\mathrm{A})$ at $\mathrm{pH} 12.2$ (aggregation inducing) and $\mathrm{pH} 7.4$ (non-aggregation inducing), excited at $370 \mathrm{~nm}$ and emission measured at 476 nm after 5 hours. B: Fluorescence microscopy images of lysozyme $(\mathrm{P})$, gelator solution $(\mathrm{G})$ and gelator with lysozyme $(\mathrm{G}+\mathrm{P})$ at $\mathrm{pH}$ 12.2. * indicates significant difference with $\mathrm{P}$ value of 0.0001 by one way ANOVA followed by post hoc Tukey's multiple comparison test

The fluorescence microscopy images of the aggregates $(\mathrm{P})$ showed the presence of small fiber-like structures, which were interconnected with each other and spread throughout the sample whereas the gelator $(1.25 \% \mathrm{w} / \mathrm{v})$ with lysozyme $(\mathrm{G}+\mathrm{P})$ showed the absence of these structures. In the control gelator sample $(G)$ these structures were also absent (Figure 2B). Further controls of protein at $\mathrm{pH} 7.4$ (no fiber-like structures) and preformed aggregated protein mixed into the gel (presence of fiber-like structures) are shown in the ESI Figure S5. These images demonstrate that the gelator formulated with the protein showed the absence of fiber like structures which were evident in the aggregates of protein and this confirms inhibition of protein aggregation. However, for these samples the gelator is predominantly in solution. Viscosity measurements showed shear thinning indicative of worm-like micelles ${ }^{39}$ (ESIFigure S10). Hence, the mechanism of inhibition of protein aggregation is probably the interaction of the amphiphilic gelator molecule with the protein as reported for other amiphiphiles ${ }^{9-10}$. Hence further evidence was required of the ability of the hydrogel fibers to inhibit protein aggregation through interaction with their hydrophobic core and another protein was selected for this purpose. Since the gelator is dissolved at $\mathrm{pH} 12.2$, we selected beta-lactoglobulin, a protein that aggregates at an acidic $\mathrm{pH}$.

The aggregation of beta-lactoglobulin was studied in $1 \mathrm{M}$ sodium chloride as previously reported. ${ }^{35}$ The $\mathrm{pH}$ was initially raised to 9 with $\mathrm{NaOH}$ and it was finally brought down to $\mathrm{pH} 4.6$ with $\mathrm{HCl}$ to initiate aggregation. The protein concentration was maintained at $3 \% \mathrm{w} / \mathrm{v}$. Firstly, different solutions of gelator without beta-lactoglobulin were prepared at concentrations from $0.15 \%$, $0.3 \%, 0.4 \%, 0.8 \%$, to $1 \% \mathrm{w} / \mathrm{v}$ with the same salt solution (ESIFigure S6A and B). All gelator concentrations formed a hydrogel stable to vial inversion and it was evident that all gels had a degree of heterogeneity where there were regions of turbidity. In addition, these regions of turbidity were found to increase with increasing
A

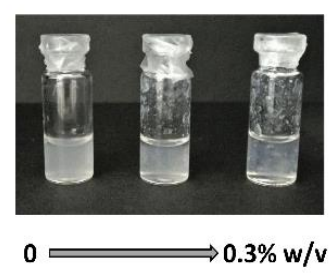

B

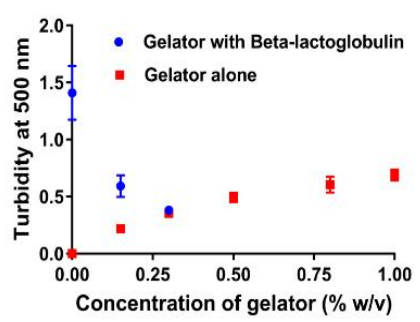

Figure 3 A: Photograph of nucleoside gelator with beta-lactoglobulin showing the intensity of cloudiness. Gelator concentration from left to right $0,0.15$ and $0.3 \% \mathrm{w} / \mathrm{v}$. B: Plot of gelator concentration versus turbidity for gelator alone and gelator with $3 \% \mathrm{w} / \mathrm{v}$ beta-lactoglobulin in $1 \mathrm{M} \mathrm{NaCl}$ at $\mathrm{pH}$ $4.6(\mathrm{I}=2 \mathrm{M})$ at different gelator concentrations after mixing at $37^{\circ} \mathrm{C}$ and allowing to cool at $22{ }^{\circ} \mathrm{C}$ for $15 \mathrm{~min}$.

gelator concentration. This is considered to be caused by precipitation of gelator which may be due to the $\mathrm{pH}$ switch (from $\mathrm{pH} 9$ to $\mathrm{pH} 5$ ) required to induce protein aggregation. The addition of betalactoglobulin was then evaluated at low gelator concentrations which displayed the least heterogeneity (ESI, Figure S6 C). These samples formed weak gels, particularly at the lowest gelator concentration of $0.15 \% \mathrm{w} / \mathrm{v}$, where water remained at the bottom of the inverted vial (ESI, Figure S6 D).

To investigate protein aggregation in this system, we therefore compared turbidity of the hydrogels and the hydrogels with protein at the same concentration of gelator. Visually, the $0.15 \% \mathrm{w} / \mathrm{v}$ gel with beta-lactoglobulin exhibited more turbidity than the gel without protein whilst for the $0.3 \% \mathrm{w} / \mathrm{v}$ sample, the turbidity was observed to be almost similar (Figure 3A). The turbidity of the gels with and without beta-lactoglobulin were then compared quantitatively using UV-Vis absorbance at $500 \mathrm{~nm}$. The turbidity data of gels without beta-lactoglobulin increased with increasing gelator concentration (Figure 3B). The turbidity of $0.15 \%$ w/v gelator sample with beta-lactoglobulin was significantly higher than that of the hydrogel without proteins whereas the turbidity of $0.3 \% \mathrm{w} / \mathrm{v}$ hydrogel with and without the protein were similar. Protein containing samples with higher gelator concentrations were not evaluated as the hydrogel itself exhibited increasing turbidity values with higher gelator concentrations. These turbidity measurements were in agreement with the visual observations (ESI-Figure S6A and B).

To further validate our observations, optical microscopy was carried out. However, from these images, it was not possible to clearly distinguish between the protein aggregates and the gel structure as the gel itself exhibited structures similar in appearance to that of protein aggregates (ESI, Figure S7).

The hydrogel's capability to inhibit protein aggregation was further verified by an ANS binding assay and fluorescence microscopy in a procedure similar to that described for lysozyme. The results of ANS fluorescence binding assay revealed a remarkable decrease in fluorescence intensity of the hydrogel containing betalactoglobulin $(\mathrm{G}+\mathrm{P})$ when compared to the solutions of protein $(\mathrm{P})$ only in the same conditions (Figure 4A). It was also evident that neither the $\mathrm{pH}$ nor the presence of the gel induced ANS fluorescence as no significant fluorescence intensity was detected in the gel alone or the solvent itself. The fluorescence microscopy images of the protein in the presence of hydrogel $(\mathrm{G}+\mathrm{P})$ did not exhibit any fiber-like interconnected structures such as those observed for the aggregated protein $(\mathrm{P})$ (Figure 4B). Further controls of protein at $\mathrm{pH} 7.4$ (no fiber-like structures) and preformed aggregated protein mixed into the gel (presence of fiber-like structures) are shown in 


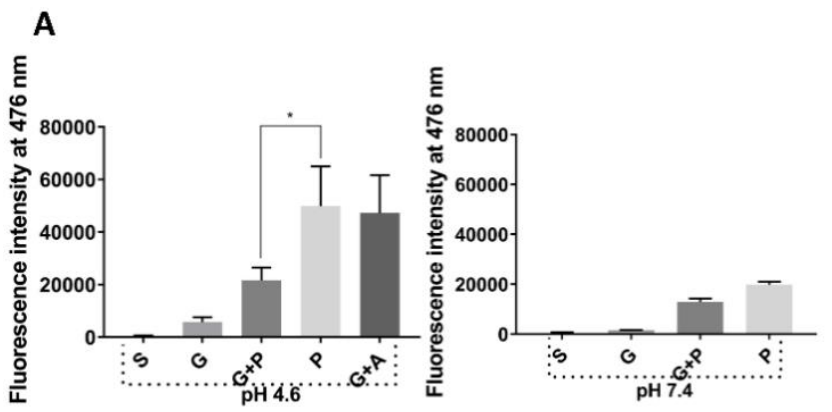

B
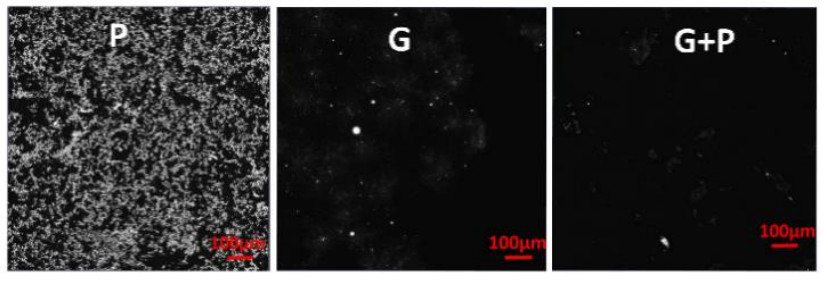

Figure 4 A: ANS Fluorescence intensities of samples and controls of beta-lactoglobulin \{ solvent $(S)$, gel $(G)$, gel with protein $(\mathrm{G}+\mathrm{P})$, protein $(\mathrm{P})$, gel with aggregates $(\mathrm{G}+\mathrm{A})\}$ at $\mathrm{pH}$ 4.6 (aggregation inducing) and $\mathrm{pH} 7.4$ (non-aggregation inducing), excited at $370 \mathrm{~nm}$ and emission measured at $476 \mathrm{~nm}$ after 5 hours. B: Fluorescence microscopy images of betalactoglobulin $(\mathrm{P})$, gel $(\mathrm{G})$, and gel with beta-lactoglobulin $(\mathrm{G}+\mathrm{P})$ at $\mathrm{pH}$ 4.6. * indicates significant difference with $\mathrm{P}$ value of 0.0001 by one way ANOVA followed by post hoc Tukey's multiple comparison test

ESI Figure S8. These data further proves our hypothesis that the gelator has the strong ability to inhibit protein aggregation. A postulation of the mechanism of inhibition of protein aggregation will now be discussed.

Since recent data from our group reports that a large amount of the gelator is in the fiber network compared to a small proportion in solution ${ }^{25}$, there is probably a significant contribution from immobilisation of the protein by the gel fiber network ${ }^{17-18}$ and a smaller contribution from the amiphiphilic gelator in solution. In general, supramolecular gels are well established as nanofiber 3D frameworks with a hydrophobic core and solvent filled cavities ${ }^{40}$ that can be used to immobilise molecules in processes such as crystillisation. ${ }^{41-42}$

Based on our experimental results we have shown that a supramolecular low molecular weight 2'-deoxycytidine based gelator exhibits the ability to suppress the aggregation of proteins with a broad range of isoelectric points (alkaline and acidic). To our knowledge, this is the first report of using a low molecular weight gelator for the inhibition of protein aggregation. Thus, the novel 2 'deoxycytidine based hydrogel has the potential to contribute to tackling one of the most important challenges in formulation of biologics, to prevent protein aggregation and improve the therapeutic efficacy of biotherapeutics. ${ }^{43}$

\section{AUTHOR INFORMATION}

\section{Corresponding Author}

Maria Marlow -School of Pharmacy, University Park Campus, University of Nottingham, NG72RD, United Kingdom.

Email:maria.marlow@nottingham.ac.uk

Mischa Zelzer-School of Pharmacy, University Park Campus, University of Nottingham, NG72RD, United Kingdom.

Email:mischa.zelzer@nottingham.ac.uk
Stephanie Allen-School of Pharmacy, University Park Campus, University of Not-tingham, NG72RD, United Kingdom.

Email:stephanie.allen@nottingham.ac.uk

Notes

The authors declare no competing financial interest.

\section{ACKNOWLEDGMENT}

This work was supported by the Engineering and Physical Sciences Research Council (EPSRC) funded CDT [grant number EP/L01646X]. Microscopy was performed using the Multidisciplinary Super Resolution Imaging Facility (School of Life Sciences) funded by Biotechnology and Biological Sciences Research Council (BBSRC) [grant number BB/L013827/1]. We thank Robert Markus for his advice and technical help.

\section{REFERENCES}

1.Roberts, C. J., Therapeutic protein aggregation: mechanisms, design, and control. Trends in biotechnology 2014, 32 (7), 372380 .

2.Fosgerau, K.; Hoffmann, T., Peptide therapeutics: current status and future directions. Drug discovery today 2015, 20 (1), 122128.

3.Uhlig, T.; Kyprianou, T.; Martinelli, F. G.; Oppici, C. A.; Heiligers, D.; Hills, D.; Calvo, X. R.; Verhaert, P., The emergence of peptides in the pharmaceutical business: From exploration to exploitation. EuPA Open Proteomics 2014, 4, 58-69. 4.Leader, B.; Baca, Q. J.; Golan, D. E., Protein therapeutics: a summary and pharmacological classification. Nature reviews Drug discovery 2008, 7 (1), 21.

5.Wang, W.; Singh, S. K.; Li, N.; Toler, M. R.; King, K. R.; Nema, S., Immunogenicity of protein aggregates - concerns and realities. International journal of pharmaceutics 2012, 431 (1-2), $1-11$.

6.Amin, S.; Barnett, G. V.; Pathak, J. A.; Roberts, C. J.; Sarangapani, P. S., Protein aggregation, particle formation, characterization \& rheology. Current Opinion in Colloid \& Interface Science 2014, 19 (5), 438-449.

7.Vagenende, V.; Yap, M. G.; Trout, B. L., Mechanisms of protein stabilization and prevention of protein aggregation by glycerol. Biochemistry 2009, 48 (46), 11084-11096.

8.Baynes, B. M.; Wang, D. I.; Trout, B. L., Role of arginine in the stabilization of proteins against aggregation. Biochemistry 2005, 44 (12), 4919-4925.

9.Kerwin, B. A., Polysorbates 20 and 80 used in the formulation of protein biotherapeutics: structure and degradation pathways. Journal of pharmaceutical sciences 2008, 97 (8), 2924-2935. 10.Baynes, B. M.; Trout, B. L., Rational design of solution additives for the prevention of protein aggregation. Biophysical journal 2004, 87 (3), 1631-1639.

11.Meyer, J. D.; Nayar, R.; Manning, M. C., Impact of bulking agents on the stability of a lyophilized monoclonal antibody. European Journal of Pharmaceutical Sciences 2009, 38 (1), 2938.

12.Challener, C., Excipient selection for protein stabilization. Pharm. Tech APIs, Excipients, and Manufacturing Supplement 2015, 39 (18), s35-s39.

13.Bondos, S. E.; Bicknell, A., Detection and prevention of protein aggregation before, during, and after purification. Analytical biochemistry 2003, 316 (2), 223-231.

14.Javid, N.; Roy, S.; Zelzer, M.; Yang, Z.; Sefcik, J.; Ulijn, R. V., Cooperative Self-Assembly of Peptide Gelators and Proteins. Biomacromolecules 2013, 14 (12), 4368-4376. 
15.Wang, H.; Wang, Z.; Yi, X.; Long, J.; Liu, J.; Yang, Z., Antidegradation of a recombinant complex protein by incoporation in small molecular hydrogels. Chemical Communications 2011, 47 (3), 955-957.

16.Conejero-Muriel, M.; Gavira, J. A.; Pineda-Molina, E.; Belsom, A.; Bradley, M.; Moral, M.; Durán, J. d. D. G.-L.; Luque González, A.; Díaz-Mochón, J. J.; Contreras-Montoya, R.; Martínez-Peragón, Á.; Cuerva, J. M.; Álvarez de Cienfuegos, L., Influence of the chirality of short peptide supramolecular hydrogels in protein crystallogenesis. Chemical Communications 2015, 51 (18), 3862-3865.

17.Kiyonaka, S.; Sada, K.; Yoshimura, I.; Shinkai, S.; Kato, N.; Hamachi, I., Semi-wet peptide/protein array using supramolecular hydrogel. Nature Materials 2003, 3, 58.

18.Scott, G.; Roy, S.; Abul-Haija, Y. M.; Fleming, S.; Bai, S.; Ulijn, R. V., Pickering Stabilized Peptide Gel Particles as Tunable Microenvironments for Biocatalysis. Langmuir 2013, 29 (46), 14321-14327.

19.Du, X.; Zhou, J.; Shi, J.; Xu, B., Supramolecular Hydrogelators and Hydrogels: From Soft Matter to Molecular Biomaterials. Chemical Reviews 2015, 115 (24), 13165-13307. 20.Li, Y.; Wang, F.; Cui, H., Peptide-based supramolecular hydrogels for delivery of biologics. Bioengineering \& Translational Medicine 2016, 1 (3), 306-322. 21.Ramin, M. A.; Sindhu, K. R.; Appavoo, A.; Oumzil, K.; Grinstaff, M. W.; Chassande, O.; Barthélémy, P., Cation tuning of supramolecular gel properties: a new paradigm for sustained drug delivery. Advanced Materials 2017, 29 (13), 1605227.

22.Tian, Y.; Wang, H.; Liu, Y.; Mao, L.; Chen, W.; Zhu, Z.; Liu, W.; Zheng, W.; Zhao, Y.; Kong, D., A peptide-based nanofibrous hydrogel as a promising DNA nanovector for optimizing the efficacy of HIV vaccine. Nano letters 2014, 14 (3), 1439-1445. 23.Koutsopoulos, S.; Zhang, S., Two-layered injectable selfassembling peptide scaffold hydrogels for long-term sustained release of human antibodies. Journal of controlled release $\mathbf{2 0 1 2}$, 160 (3), 451-458.

24.Koutsopoulos, S.; Unsworth, L. D.; Nagai, Y.; Zhang, S., Controlled release of functional proteins through designer selfassembling peptide nanofiber hydrogel scaffold. Proceedings of the National Academy of Sciences 2009, 106 (12), 4623-4628. 25.Angelerou, M.; Frederix, P. W.; Wallace, M.; Yang, B.; Rodger, A.; Adams, D. J.; Marlow, M.; Zelzer, M., A supramolecular nucleoside-based gel: Molecular dynamics simulation and characterization of its nanoarchitecture and selfassembly mechanism. Langmuir 2018, 6912-6920 26.Skilling, K. J.; Kellam, B.; Ashford, M.; Bradshaw, T. D.; Marlow, M., Developing a self-healing supramolecular nucleoside hydrogel. Soft matter 2016, 12 (43), 8950-8957.

27.Vermonden, T.; Censi, R.; Hennink, W. E., Hydrogels for protein delivery. Chemical Reviews 2012, 112 (5), 2853-2888. 28.Li, Q.; Liu, C.; Wen, J.; Wu, Y.; Shan, Y.; Liao, J., The design, mechanism and biomedical application of self-healing hydrogels. Chinese Chemical Letters 2017, 28 (9), 1857-1874.
29.Cao, S.; Fu, X.; Wang, N.; Wang, H.; Yang, Y., Release behavior of salicylic acid in supramolecular hydrogels formed by 1-phenylalanine derivatives as hydrogelator. International journal of pharmaceutics 2008, 357 (1-2), 95-99.

30.Skilling, K. J.; Ndungu, A.; Kellam, B.; Ashford, M.; Bradshaw, T. D.; Marlow, M., Gelation properties of selfassembling $\mathrm{N}$-acyl modified cytidine derivatives. J.Mater.Chem 2014, 2 (47), 8412-8417.

31.Swaminathan, R.; Ravi, V. K.; Kumar, S.; Kumar, M. V. S.; Chandra, N., Lysozyme: a model protein for amyloid research. In Advances in protein chemistry and structural biology, Elsevier: 2011; Vol. 84, pp 63-111.

32.Wang, W., Protein aggregation and its inhibition in biopharmaceutics. International journal of pharmaceutics $\mathbf{2 0 0 5}$, 289 (1-2), 1-30.

33.Novák, P.; Havlíček, V., Protein Extraction and Precipitation. In Proteomic Profiling and Analytical Chemistry (Second Edition), Elsevier: 2016; pp 51-62.

34.Kumar, S.; Ravi, V. K.; Swaminathan, R., Suppression of lysozyme aggregation at alkaline $\mathrm{pH}$ by tri-N-acetylchitotriose. Biochimica et Biophysica Acta (BBA)-Proteins and Proteomics 2009, 1794 (6), 913-920.

35.Majhi, P. R.; Ganta, R. R.; Vanam, R. P.; Seyrek, E.; Giger, K.; Dubin, P. L., Electrostatically driven protein aggregation: $\beta$ lactoglobulin at low ionic strength. Langmuir 2006, 22 (22), 9150-9159.

36.von Sonntag, C., Nucleobases, Nucleosides and Nucleotides. Free-Radical-Induced DNA Damage and Its Repair: A Chemical Perspective 2006, 211-334.

37.Sato, T.; Mattison, K. W.; Dubin, P. L.; Kamachi, M.; Morishima, Y., Effect of protein aggregation on the binding of lysozyme to pyrene-labeled polyanions. Langmuir 1998, 14 (19), 5430-5437.

38.Rajan, R.; Matsumura, K., Inhibition of protein aggregation by zwitterionic polymer-based core-shell nanogels. Scientific reports 2017, 7, 45777.

39.Castilla, A. M.; Wallace, M.; Mears, L. L. E.; Draper, E. R.; Doutch, J.; Rogers, S.; Adams, D. J., On the syneresis of an OPV functionalised dipeptide hydrogel. Soft Matter 2016, 12 (37), 7848-7854.

40.Foster, J. A.; Steed, J. W., Exploiting Cavities in Supramolecular Gels. Angewandte Chemie International Edition 2010, 49 (38), 6718-6724.

41.Foster, J. A.; Piepenbrock, M. O. M.; Lloyd, G. O.; Clarke, N.; Howard, J. A. K.; Steed, J. W., Anion-switchable supramolecular gels for controlling pharmaceutical crystal growth. Nature Chemistry 2010, 2 (12), 1037-1043.

42. Kaufmann, L.; Kennedy, S. R.; Jones, C. D.; Steed, J. W., Cavity-containing supramolecular gels as a crystallization tool for hydrophobic pharmaceuticals. Chemical Communications 2016, 52 (66), 10113-10116.

43. Drury, J. L.; Mooney, D. J., Hydrogels for tissue engineering: scaffold design variables and applications. Biomaterials 2003, 24 (24), 4337-4351. 